\title{
As dificuldades de aprendizagem discente e as tecnologias educacionais em tempos de pandemia de COVID-19 ${ }^{1}$
}

\author{
Student learning difficulties and educational technologies in times of the COVID- \\ 19 pandemic
}

\section{Dificultades de aprendizaje de los estudiantes y tecnologías educativas en tiempos de pandemia del COVID-19}

\author{
Maria Neuraildes Gomes Viana ${ }^{2 *}$, Walter Rodrigues Marques $1^{3}$, Tassiane Sousa Correa \\ Branco $^{4}$, Frederic Menezes Ferreira3 ${ }^{5}$, Maria do Socorro Estrela Paixão4 ${ }^{5}$
}

\begin{abstract}
RESUMO
$\mathrm{O}$ artigo reflete sobre os aspectos referentes aos problemas de aprendizagem junto à prática do professor, onde este se sente desafiado a repensar a prática pedagógica, inscrevendo a possibilidade de novos procedimentos. $\mathrm{O}$ uso das tecnologias ainda se apresenta como um desafio para muitos professores no desenvolvimento do trabalho docente. Questionamentos e reflexões sobre quais são os reais potenciais educativos das tecnologias para o processo ensino e aprendizagem, e de que forma elas podem influenciar as ações pedagógicas, estão presentes nas pesquisas educacionais. O artigo discute o uso das novas tecnologias no processo de ensino-aprendizagem no âmbito escolar. A partir de pesquisa bibliográfica, verifica-se a inserção das tecnologias, compreendendo os impactos da sua utilização nas atividades de professores e na apropriação pelos alunos. O objetivo do artigo busca analisar possíveis dificuldades encontradas por docentes e discentes a partir da utilização das tecnologias no processo educativo. Para sustentar as hipóteses levantadas procurou-se respaldo teórico em bibliografia especializada. A escolha do tema teve como intuito investigar quais dificuldades há na inserção das tecnologias nos espaços escolares e como irão se comportar os alunos na cultura digital em tempos de pandemia. As dificuldades de aprendizagem têm causas variadas. Entretanto, parte -se do pressuposto de que as dificuldades dos discentes em utilizar as tecnologias poderiam ser minimizadas se escolas e professores estivessem preparados para lidar com tal situação. Desta forma, entende-se que esta pesquisa possa contribuir de maneira para a reflexão de educadores preocupados com o desempenho dos educandos.
\end{abstract}

Palavras-chave: Educação; Ensino Aprendizagem; Docentes; Discentes; Novas Tecnologias.

\footnotetext{
ABSTRACT

The article reflects on the aspects related to learning problems with the teacher's practice, where he feels challenged to rethink the pedagogical practice, inscribing the possibility of new procedures. The use of technologies is still a challenge for many teachers in the development of teaching work. Questions and reflections on what are the real educational potentials of technologies for the teaching and learning process, and how they can influence pedagogical actions, are present in educational research. The article discusses the use of new technologies in the teaching-learning process at school. From bibliographic research, the insertion of technologies is verified, understanding the impacts of their use in the activities of teachers and in the appropriation by students. The aim of the article seeks to analyze possible difficulties encountered by teachers and students from the use of technologies in the educational process. To support the hypotheses raised, theoretical support was sought in specialized bibliography. The choice of the theme was intended to

${ }^{1}$ Artigo apresentado no Simpósio Internacional e Nacional de Tecnologias Digitais na Educação

${ }^{2}$ Secretaria Municipal de Educação de Bacabeira - MA. *E-mail: neura.gomes@hotmail.com

${ }^{3}$ Secretaria de Estado da Educação do Maranhão

${ }^{4}$ Centro Universitário do Maranhão

${ }^{5}$ Universidade Federal do Maranhão
} 
investigate what difficulties there are in the insertion of technologies in school spaces and how students will behave in digital culture in times of pandemic. Learning difficulties have different causes. However, it is assumed that the difficulties of students in using technologies could be minimized if schools and teachers were prepared to deal with this situation. Thus, it is understood that this research can contribute in a way to the reflection of educators concerned with the performance of students.

Keywords: Education; Teaching Learning; Teachers; Students; New technologies.

\section{RESUMEN}

El artículo reflexiona sobre los aspectos relacionados con los problemas de aprendizaje con la práctica del docente, donde se siente desafiado a repensar la práctica pedagógica, inscribiendo la posibilidad de nuevos procedimientos. El uso de tecnologías sigue siendo un desafío para muchos docentes en el desarrollo de la labor docente. Las preguntas y reflexiones sobre cuáles son los verdaderos potenciales educativos de las tecnologías para el proceso de enseñanza y aprendizaje, y cómo pueden influir en las acciones pedagógicas, están presentes en la investigación educativa. El artículo analiza el uso de las nuevas tecnologías en el proceso de enseñanza-aprendizaje en la escuela. A partir de la investigación bibliográfica se verifica la inserción de tecnologías, entendiendo los impactos de su uso en las actividades de los docentes y en la apropiación por parte de los estudiantes. El objetivo del artículo busca analizar las posibles dificultades encontradas por docentes y estudiantes por el uso de tecnologías en el proceso educativo. Para apoyar las hipótesis planteadas, se buscó apoyo teórico en bibliografía especializada. La elección de la temática pretendía indagar qué dificultades hay en la inserción de tecnologías en los espacios escolares y cómo se comportarán los estudiantes en la cultura digital en tiempos de pandemia. Las dificultades de aprendizaje tienen diferentes causas. Sin embargo, se asume que las dificultades de los estudiantes en el uso de tecnologías podrían minimizarse si las escuelas y los profesores estuvieran preparados para enfrentar esta situación. Así, se entiende que esta investigación puede contribuir de alguna manera a la reflexión de los educadores preocupados por el desempeño de los estudiantes.

Palabras clave: Educación; Enseñanza del aprendizaje; Maestros; Estudiantes; Nuevas tecnologías.

\section{INTRODUÇÃO}

O artigo reflete sobre a realidade escolar quanto ao uso das TIC (Tecnologias da Informação e Comunicação), mostrando quão difícil está sendo as escolas adaptarem a cultura digital em tempos de pandemia. $\mathrm{O}$ termo tecnologia refere-se ao conjunto de conhecimentos, especialmente princípios científicos, que se aplicam a um determinado ramo de atividade, ou seja, o vocabulário peculiar de uma ciência. (FERREIRA, 1999). Marques et al. (2020, p. 66050) definem tecnologia como sendo "[...] de origem grega (tékhne + logos), cujo significado, segundo o Dicionário Aurélio (2001) é o "conjunto de conhecimento, especialmente princípios científicos, que se aplicam a um determinado ramo de atividade". Os autores afirmam ainda que:

A tecnologia surge quando há dificuldade, podemos relacionar essa afirmação nos referindo à pré-história quando o homem começou a produzir seus 
primeiros instrumentos para facilitar suas atividades do cotidiano. Utilizando incialmente a pedra como matéria prima e depois osso, madeira, marfim. Com o passar do tempo mulheres e homens criam equipamento mais elaborados, ampliando suas utilidades que vão desde a agricultura, passando pela ciência e chega até na Arte (MARQUES et al., 2020, p. 66050).

As Tecnologias da Informação e Comunicação vêm se desenvolvendo e se incorporando à vida cotidiana de forma acelerada. Quando aplicadas à educação, agregam-se ao processo ensino-aprendizagem como mídias de grandes potenciais pedagógicos. Para tal, existe a necessidade de acompanhar e responder às demandas e desafios postos pela sociedade do conhecimento por meio da tecnologia, pois sabe-se que as tecnologias favorecem o aprendizado individualizado, coletivo e em rede.

$\mathrm{Na}$ educação, o foco, além de ensinar, é ajudar a integrar o ensino à vida, conhecimento e ética, reflexão e ação, a ter uma visão da totalidade. Educar é ajudar a integrar todas as dimensões da vida, é ajudar a encontrar o caminho intelectual, emocional, profissional e que contribua para modificar a sociedade. O avanço tecnológico marca profundamente o mundo contemporâneo e os cenários educativos, a tecnologia já está presente em todos os avanços e de diversas formas. O seu rápido avanço nas últimas décadas criou um mundo novo e novas maneiras de viver.

Tecnologia e educação caminham juntas e são mecanismos que auxiliam na formação do indivíduo, disponibilizando e sistematizando informações no processo de comunicação e interação, servindo como base de apoio, possibilitando e conduzindo para a estruturação ou construção/reconstrução dos conhecimentos.

A tecnologia educacional passou a ser compreendida como uma opção de se fazer educação contextualizada com as questões sociais e suas contradições, visando o desenvolvimento integral do homem e sua inserção crítica no mundo.

Pucci (2003) discute que a tecnologia se faz presente na vida das pessoas em todos os ambientes, seja no interior de suas casas, nas ruas, nas salas de aula, onde estiverem os aparelhos tecnológicos direcionando as atividades, condicionando o modo de pensar, relacionar e agir das pessoas. De acordo com o autor, a comunicação é um processo que ocorre não somente na forma do encontro físico, mas, sobretudo, por conta das longas distâncias, através dos meios tecnológicos, ou seja, quem é responsável por essa supressão é a tecnologia, capaz de construir interações em diferentes situações.

Segundo Brito e Purificação (2008, p.23) “[...] estamos em um mundo em que as tecnologias interferem no cotidiano, sendo relevante, assim, que a educação também envolva a democratização do acesso ao conhecimento, à produção e à interpretação das 
tecnologias”. Segundo Castells (2006), as mudanças proporcionadas pelas tecnologias estão presentes em todas as estruturas da sociedade, bem como as suas aplicações e consequências. No âmbito da educação, isso não é diferente, pois está presente dentro e fora da escola e da universidade por meio da cultura contemporânea.

O artigo busca responder à pergunta: quais dificuldades há na inserção das tecnologias nas salas de aula em tempos de pandemia? E tem por objetivo analisar as possíveis dificuldades encontradas por docente e discentes a partir da utilização das tecnologias no processo educativo durante a pandemia, bem como identificar os principais problemas enfrentados pelos docentes na utilização destas ferramentas; verificar se os discentes usam as tecnologias a favor de sua aprendizagem; verificar o principal desafio do professor no ensino aprendizagem dos alunos com as TIC.

A problemática central do estudo baseia-se nestas indagações e na ausência de conhecimento e aprofundamento sobre a compreensão dessas ferramentas entre docentes e discentes. O trabalho busca fazer com que os professores reflitam e percebam quais dificuldades há na inserção das tecnologias e a importância disso na atualidade. Dessa forma, busca-se fazer com que repensem a forma de usá-las.

No decorrer dos estudos apresenta-se reflexões referentes à tecnologia no contexto educacional; a postura pedagógica assumida pelo professor frente às tecnologias e a contribuição desses recursos tecnológicos frente à pandemia para um ensino de qualidade.

\section{REFERENCIAL TEÓRICO}

Um dos maiores desafios da escola contemporânea é aprender a lidar com a tecnologia e transformá-la em aliada da educação. Os professores foram, são e continuarão sendo mediadores indispensáveis no aprendizado, o que não descarta a necessidade de aprender a lidar com a tecnologia. É preciso transformar as ferramentas tecnológicas em potencializadoras do ensino-aprendizado. Além disso, elas devem ser usadas como meio e não como fim na educação.

O uso das tecnologias ainda se apresenta como um desafio para muitos professores no desenvolvimento do trabalho docente. Questionamentos e reflexões sobre quais são os reais potenciais educativos das tecnologias para o processo ensino e aprendizagem, e de que forma elas podem influenciar as ações pedagógicas, estão presentes nas pesquisas educacionais. Neste sentido, este artigo tem como objetivo apresentar dificuldades 
apontadas por parte dos professores no uso das Tecnologias da Informação e Comunicação no trabalho docente.

O professor precisa vencer o receio de usar as tecnologias em seu trabalho docente e terá que ser responsável por esta ruptura paradigmática a partir da mudança do próprio comportamento. Pois, somente mediante esta mudança as dificuldades aqui apresentadas e muitas outras que possam surgir serão superadas permitindo a utilização dos potenciais educativos das TIC.

Para Peixoto, Brandão e Santos (2007), o sentido mais amplo da tecnologia não se refere só à sua utilidade funcional. É preciso ter em mente que a tecnologia ao servir a determinada prática pedagógica, os sujeitos envolvidos experimentem a tecnologia de modo a incorporá-la. Isso significa que tanto professores e alunos precisam se apropriar das TIC de forma que sua utilização e a construção do conhecimento se efetuem como cocriação e não simplesmente como transmissão. Para tanto, o docente precisa atuar com base em um novo paradigma, não mais como apenas transmissor de informação, mas na criação de situações de aprendizagem nas quais o aluno realiza atividades e constrói o seu conhecimento.

Percebe-se que o uso das tecnologias no trabalho docente exige concepções e metodologias de ensino diferentes das tradicionais, para atender as necessidades educacionais contemporâneas. Portanto, é necessário que os professores desenvolvam um debate sobre a relevância das tecnologias no trabalho docente e sobre a melhor maneira de usá-las, para que não sejam vistas e trabalhadas como um recurso meramente técnico.

Moran (2006) afirma que em geral os professores têm dificuldades no domínio das tecnologias e, tentam fazer o máximo que podem, diante deste hábito mantêm uma estrutura repressiva, controladora, repetidora. Muitos tentam mudar, mas não sabem bem como fazê-lo e não se sentem preparados para experimentar com segurança. Ainda segundo Moran (2006, p. 32), “[...] é importante diversificar as formas de dar aula, de realizar atividades e de avaliar".

Porém, é importante ressaltar que é preciso mais do que um simples domínio instrumental, tornando-se necessário um conhecimento das potencialidades proporcionadas por cada tipo de tecnologia de acordo com cada método de ensino a ser aplicado.

Segundo Almeida (2001, p. 43), o professor ao incorporar as TIC aos métodos ativos de aprendizagem, "além de desenvolver a habilidade de uso das mesmas, estabelece uma ligação entre esse domínio, a prática pedagógica, as teorias educacionais 
refletindo sobre sua própria prática buscando transformá-la". Várias instituições de acordo com Moran (2006), exigem mudanças dos professores sem lhes dar condições para que eles as efetuem. Muitas das organizações introduzem computadores, conectam com a Internet e esperam que só isso melhore o processo educativo.

Neste sentido, o objetivo é fazer uma reflexão acerca do papel do professor destacando os desafios que as tecnologias estão impondo aos profissionais da educação para torná-los em um profissional ainda mais competente na contemporaneidade, refletir também, sobre as contribuições das tecnologias na construção de novos caminhos para ensinar e aprender na sociedade contemporânea.

Segundo Miranda (2007) de fato o uso das tecnologias exige um esforço de reflexão e de modificação de concepções e práticas de ensino, que a maioria dos professores não está disponível para fazer. E não será tarefa fácil, pois é preciso esforço, persistência e empenho.

Kenski (2009, p.103) afirma que:

\begin{abstract}
Um dos grandes desafios que os professores brasileiros enfrentam está na necessidade de saber lidar pedagogicamente com alunos e situações extremas: dos alunos que já possuem conhecimentos avançados e acesso pleno às últimas inovações tecnológicas aos que se encontram em plena exclusão tecnológica; das instituições de ensino equipadas com mais modernas tecnologias digitais aos espaços educacionais precários e com recursos mínimos para o exercício da função docente. $\mathrm{O}$ desafio maior, no entanto, ainda se encontra na própria formação profissional para enfrentar esses e tantos outros problemas.
\end{abstract}

Perrenoud (2000) afirma que as competências não são apenas saberes, mas integram, incorporam conhecimentos adquiridos no decorrer da história de vida da pessoa. Uma ideia comum na nossa cultura é a diferença entre saber e saber fazer. Não basta saber, sendo necessário saber aplicar, mobilizar, pôr em movimento, transformar em ação visível.

Segundo Correia (2007) o professor na realização do trabalho docente estará sempre diante de situações complexas para as quais precisar ir buscar respostas, muitas das vezes podem ser até repetitivas e outras vezes criativas, que dependerão de sua capacidade e habilidade de leitura da realidade e do contexto em que ele estiver inserido. $\mathrm{Na}$ sua formação profissional precisa se preparar para enfrentar o cotidiano imprevisível da sala de aula.

Verifica-se que é necessário que o professor supere o método da transmissão de conhecimentos e busque um ensino mais contextualizado, adequado às exigências contemporâneas no mundo do trabalho. Além disso, é necessário que os professores se 
apropriem das tecnologias, desenvolvendo alternativas educacionais apropriadas em prol do aprendizado, e da agregação do conhecimento e da motivação de seus alunos. Conhecer essas tecnologias é uma necessidade diretamente ligada à eficácia do ensino.

Valente (2008) salienta que para o professor utilizar as tecnologias em sala de aula, ele necessita ter um conhecimento técnico básico para manejar todos os recursos tecnológicos que podem ser utilizados pedagogicamente. Por isso, é necessário que o professor invista na aquisição do conhecimento tecnológico.

Diante do exposto, verifica-se que os desafios impostos aos docentes na contemporaneidade giram em torno das ferramentas tecnológicas disponíveis. Um dos exemplos que podemos citar é a própria internet, em que os alunos terão a oportunidade de se comunicar com muitos receptores, permitindo a troca de informações e o desenvolvimento do senso crítico, podendo contribuir para aprimorar a escrita e o pensamento crítico. É importante pensar numa educação com objetivos mais amplos, numa perspectiva de ação visando à busca de valores comprometidos com uma sociedade mais humana e com mais justiça social.

O desenvolvimento tecnológico é uma das transformações que revolucionariam todas as áreas, principalmente, as que lidam com o conhecimento. Nossa sociedade utiliza as novas tecnologias em larga escala, "todas elas como ferramentas que auxiliam as pessoas a viverem melhor dentro de um determinado contexto social e espaço temporal" (ALVES; NOVA, 2003, p. 25), causando profundas mudanças que proporcionam facilidades e progressos, especialmente, no que se refere às tecnologias da informação e da comunicação.

A utilização das novas tecnologias na educação deve estar fundamentada nas concepções de ensinar e aprender, diferentes das propostas nos modelos curriculares notadamente tradicionais e defasadas. A tecnologia aliada à educação, proporciona aos alunos o acesso às informações de forma interessante, e dinâmica de estratégias de aprender bem.

Portanto, a atividade educativa, como processo de comunicação, deve ter o feedback entre docente e discente. O diálogo consubstancia, assim, a otimização do ato educativo, enriquecendo sua atividade de aprendizagem.

Takadashi (2000), enfatiza que:

Pensar educação na sociedade da informação exige considerar um leque de aspectos relativos às tecnologias de informação e comunicação, a começar pelo 
papel que elas desempenham na construção de uma sociedade que tenha a inclusão e a justiça social como uma das prioridades principais.

Surge então no cenário, principal educacional, a proliferação das redes de comunicação digital, que traz inúmeras possibilidades e perspectivas de inovação tecnológica. A chamada Revolução Digital refere-se à criação de grandes sistemas de redes de comunicação integradas, com suporte de armazenamento e transmissão de dados digitais.

Neste cenário, verifica-se que a evolução tecnológica vem contribuindo para o estabelecimento de uma nova ordem econômica e social nos mais variados campos da ação humana, essencialmente na área da educação

As tecnologias da informação e da comunicação se convertem em tecnologias educativas na medida em que são utilizadas como estratégias de ensino aprendizagem, e não como meros recursos de demonstração. Portanto, os diversos meios não são, a priori, tecnologias educativas, mas podem vir a ser qualificadas para tais funções.

As NTIC oferecem possibilidades inéditas e imensuráveis de interação e interatividade mediatizada. Essas possibilidades apresentam grandes vantagens em permitir a combinação da flexibilidade da interação humana com a independência no tempo e no espaço.

Dentre os principais recursos tecnológicos mais importante para essa crise de pandemia é o Google Meet, o qual se parece muito com a videoconferência, pois é uma tecnologia que permite que grupos distantes, situados em dois ou mais lugares geograficamente diferentes, comuniquem-se "ace a face", através de sinais de áudio e vídeo, à distância, as condições de um encontro entre pessoas. A plataforma Google Meet e a que mais se aproxima de uma situação convencional da sala de aula, possibilita a conversa em duas vias, permitindo que o processo de ensino aprendizagem ocorra em tempo real (online) e possa ser interativo, entre pessoas que podem se ver e ouvir simultaneamente. É um recurso tecnológico muito utilizado atualmente.

Conforme podemos observar, o seu uso no contexto educativo adquiriu uma importância incomensurável, pois proporciona oportunidades de inovação do processo ensino-aprendizagem, cria de novos espaços e tempos educacionais.

Como confirma Takadashi (2000), “[...] as tecnologias de informação e comunicação devem ser utilizadas para integrar a escola e a comunidade, com a sorte de que a educação mobilize a sociedade”. A exigência da atual realidade, firma-se no desenvolvimento de um sujeito preparado para agir e interagir onde a tecnologia se faz 
presente. Este sujeito deve ser capaz de adaptar-se as novas realidades, apresentando disponibilidade e capacidade para aprendizagem

De acordo com Kenski:

\begin{abstract}
$\mathrm{Na}$ era da informação, comportamentos, práticas, informações e saberes se alteram com extrema velocidade. Um saber ampliado e mutante caracteriza o estágio do conhecimento na atualidade. Essas alterações refletem-se sobre as tradicionais formas de ensinar e aprender possibilitadas pela atualidade tecnológica. Abrir-se para novas educações, resultantes de mudanças estruturais de ensinar e aprender possibilitadas pela atualidade tecnológica, é o desafio a ser assumido por toda sociedade. (KENSKI, 2012, p. 41).
\end{abstract}

Para Demo (2009, p.63) "[...] novas tecnologias fazem parte das novas alfabetizações, das habilidades do século XXI, tornando-se [...] parte fundamental”. Não se trata apenas de se utilizar das tecnologias para o trabalho e/ou repasse de conceitos e conhecimentos científicos, mas de tomar consciência que por meio delas, podemos expandir os espaços de aprendizagem, ampliando as possibilidades de leitura e expressão da realidade. A tecnologia se torna importante no momento que propicia ao professor a realização de novas práticas pedagógicas, associando a um novo recurso tecnológico, gerando possibilidades de interação e construção do conhecimento.

Para Kenski (2012) a maioria das tecnologias é utilizada com a finalidade de auxiliar o processo educativo e, dependendo da tecnologia escolhida para o desenvolvimento da aula, pode haver mudanças no processo educacional, alterando a forma de comunicação entre os participantes.

A utilização dos recursos tecnológicos deve considerar o contexto educativo na totalidade, não somente as mudanças proporcionadas na sala de aula, na maneira como o professor ensina e na aprendizagem do aluno, mas considerar a escola como um todo.

De acordo com Vosgerau:

[...] se realmente queremos que as tecnologias representem benefícios na aprendizagem e na vida dos alunos, temos de começar a enxergar a escola como um todo, analisar as possibilidades, os limites, os entraves para escola se tornar realmente um espaço de inclusão social e digital, levando de fato nossas crianças e jovens a aprender mais e melhor. (VOSGERAU, 2011, p. 37).

A escola tem o papel de desenvolver a análise crítica de seus alunos e professores, a tomada de consciência em relação ao poder de escolha, para que os mesmos não se transformem em consumidores em potencial. Possibilitar ao aluno um ensino coerente com a realidade existente, ser capaz de fazer a leitura de mundo, não somente a leitura da 
palavra, ou seja, levar o aluno a interpretações do contexto em que está inserido para que, de maneira crítica, realize suas escolhas e defina suas necessidades é função da escola.

A reflexão sobre o uso dos recursos tecnológicos no processo ensino aprendizagem de maneira crítica e responsável é fundamental para a apropriação de conceitos e conhecimentos. Estes contribuem para a melhoria da prática pedagógica do professor, traduzindo-se numa aprendizagem significativa para o aluno.

Considerando que a leitura crítica da comunicação, por meio de suas atividades pedagógicas, permite, com base em reflexões sistemáticas, a formação de leitores críticos não só dos meios de comunicação de massa como do próprio mundo, a utilização dos recursos tecnológicos e suas linguagens devem favorecer a expressão dos indivíduos.

Portanto, refletir sobre o uso dos recursos tecnológicos no processo ensino aprendizagem de maneira crítica e responsável é fundamental para a apropriação de conceitos e conhecimentos que contribuam para a melhoria da prática pedagógica do professor traduzindo-se numa aprendizagem significativa para o aluno.

Não é só por parte dos docentes que deve haver uma adequação a essa sociedade mais tecnológica, mas também a todos aqueles que agem como corresponsáveis por uma educação de mais qualidade e contextualizada com o tempo em que se está inserido.

Segundo Nóvoa (2001), para atender às novas exigências da contemporaneidade, o professor necessita de atualizações constantes. A formação inicial é apenas o primeiro passo de uma caminhada, o professor precisa participar constantemente de capacitações individuais e coletivas, para acompanhar as evoluções e atender a complexidade das salas de aula na atualidade.

Os professores devem oferecer aos alunos a possibilidade de adquirir o conhecimento e de praticá-lo num contexto de uso o mais realista possível. E que exista reciprocidade, ou seja, quando exista um laço de comunicação forte entre quem aprende e quem ensina, para que ocorra uma aprendizagem mais efetiva

\section{METODOLOGIA}

A pesquisa é bibliográfica, qualitativa e buscou ancorar-se teoricamente em: Kenski (2009, 2012); Nóvoa (2001); Takadashi (2000); Vosgerau (2011) e outros. Primeiramente, foi realizado um levantamento bibliográfico sobre a situação educacional em tempos de pandemia e em seguida, buscou-se inter-relacionar tais referências bibliográficas com as problemáticas vividas na escola tanto por professores quanto por 
alunos. Objetivou responder ao questionamento sobre que influências a inserção de tecnologias nesse período exerceria sobre os dois lados da educação [professores e alunos], buscando analisar as perdas e ganhos no processo ensino-aprendizagem nesse contexto pandêmico.

\section{CONSIDERAÇÕES FINAIS}

Quando manifesta dificuldades, o aluno revela uma situação mais ampla, em que também se inscreve a escola, uma vez que esta é parceria no processo da aprendizagem. Nessa parceria entra o professor, e cabe à escola, abrir espaços para que se disponibilizem recursos que façam frente aos desafios, isto é, na direção da efetivação da aprendizagem. Nesse sentido, o professor sente-se desafiado a repensar a prática pedagógica, inscrevendo a possibilidade de novos procedimentos. Esse processo de parceria possibilita uma aprendizagem muito importante e enriquecedora. Para sermos bons profissionais hoje, necessitamos crescer profissionalmente, estar atentos as mudanças estar sempre se atualizando.

Fica claro que as dificuldades de aprendizagem são causadas por diversos fatores, entretanto, entende-se que os discentes com dificuldades em utilizar as tecnologias poderiam seguir sem maiores conflitos desde que encontrassem escolas e professores bem-preparados para tal situação.

Porém são diversas as dificuldades encontradas, necessitam então, de mais prioridade e investimento por parte do governo.

Portanto, antes de qualquer posição em relação às dificuldades de aprendizagem, o educador ou a instituição de ensino deve antes de tudo fazer uma reflexão da realidade escolar quanto ao uso das tecnologias, mostrando assim quão difícil está sendo as escolas adaptarem a cultura digital nessa crise de pandemia.

Segundo os Padrões de Competência da UNESCO (2009), as práticas educacionais tradicionais já não oferecem aos futuros professores todas as habilidades necessárias para capacitar os alunos a sobreviverem no atual mercado de trabalho. Logo, estes padrões de competência reforçam que os professores que estão em exercício necessitam estar preparados para utilizar as tecnologias e para ofertar autonomia a seus alunos. O professor na contemporaneidade necessita ser capaz de saber quais as vantagens e as oportunidades de aprendizagem que estas tecnologias podem proporcionar a seus alunos. As salas de aula, tanto presenciais quanto virtuais, necessitam de professores com habilidades em tecnologia que permitam realmente transmitir o conhecimento ao mesmo 
tempo que se incorporam conceitos e competências em tecnologia da informação e comunicação.

As tecnologias causaram enormes benefícios em termos de avanço científico, educação, comunicação, lazer, processamento de dados, busca e produção do conhecimento. Assim sendo, se constituem como molas propulsoras e recursos dinâmicos para favorecer o trabalho docente, à medida que, quando bem utilizadas pelos professores e alunos permitem intensificar a melhoria das práticas pedagógicas desenvolvidas em sala de aula e fora dela.

Portanto, conclui se que o professor precisa vencer o receio de usar as tecnologias em seu trabalho docente e terá que ser responsável por esta ruptura paradigmática a partir da mudança do próprio comportamento. Pois, somente mediante esta mudança as dificuldades aqui apresentadas e muitas outras que possam surgir serão superadas permitindo a utilização dos potenciais educativos das TIC.

Conclui-se que se faz importante tanto para o professor como para o aluno o acesso as informações durante o período dessa pandemia, tanto na relação como comunicação quanto para o estímulo as habilidades escolares. Os docentes podem encontrarem inúmeros recursos que facilitam a didática, materiais que instigam o aprendizado e sem contar a possibilidade que os alunos tenham acesso em tempo real ao material, independente do lugar em que se encontram.

\section{REFERÊNCIAS}

ALMEIDA, Maria. Elizabeth. B. de. Educação, projetos, tecnologia e conhecimento. São Paulo: PROEM, 2001. 63p.

ALVES, L.; NOVA, C. (Org.). Educação a distância: uma nova concepção de aprendizagem e interatividade. São Paulo: Futura, 2003.

BRITO, G. da S; PURIFICAÇÃO, I. da. Educação e novas tecnologias: um repensar. Curitiba - PR: Ipbex, 2008.

CORREIA, C. C. Um programa de professores em informática educativa como espaço para inovações tecnológicas na prática docente Rio de Janeiro. 2007. $120 \mathrm{f}$. Universidade Estadual de Sá, Rio de Janeiro, 2007.

DEMO, Pedro. Educação hoje: "Novas" tecnologias, pressões e oportunidades. São Paulo: Atlas, 2009.

FERREIRA, Aurélio Buarque de Holanda. Dicionário eletrônico: século XXI. Rio de Janeiro: Nova Fronteira / Lexion Informática, 1999. 1 CD-ROM. Versão 3.0. 
KENSKI, Vani Moreira. Tecnologias e Ensino presencial e a Distância. 2. ed. Campinas, SP: Papirus, 2009

KENSKI, V. M. Educação e tecnologias: o novo ritmo da informação. 5. ed. Campinas, SP: Papirus, 2012. 141p.

MARQUES, Walter Rodrigues; ROCHA, Viviane Moura da; ROCHA, Luís Félix de Barros Vieira; MARQUES, Ana Paula Cerqueira; CANTANHEDE, Meiryele Coelho. Aplicabilidade da tecnologia no ensino e na produção artística. Braz. J. of Develop., Curitiba, v. 6, n. 9, p. 66049-66058, set. 2020. Disponível em: <https://www.brazilianjournals.com/index.php/BRJD/article/view/16275/13304>.

Acesso em: 14 fev. 2022.

MIRANDA, G. L. Limites e possibilidades das TIC na educação. 2007.

MORAIS, Maria. Cândida. Blog: Educação e Tecnologia. Disponível em: $<$ http://estudandoeducacaoetecnologia.blogspot.com>. Acesso em: 22 jul. 2020.

MORAN, José Manoel. Mudar a forma de ensinar e de aprender com tecnologias: transformar as aulas em pesquisa e comunicação presencial-virtual. INTERAÇÕES

v. 5, n. 9, p. 57-72, jan./jun. 2000, São Paulo. Disponível em: <https://www.redalyc.org/pdf/354/35450905.pdf>. Acesso em: 10 set. 2020.

MORAN, José Manoel. Novas tecnologias e mediação pedagógica. 6. ed. Campinas, SP: Papirus. 2006, p. 1166.

MORAN, José Manoel. A educação que desejamos: Novos desafios e como chegar lá. Campinas, SP: Papirus, 2007

NÓVOA, Antônio. Professor pesquisador e reflexivo. Rio de Janeiro, 13 set. 2001.

PEIXOTO, M. de A. P.; BRANDÃO, M. A. G. B.; SANTOS, G. dos. Metacognição e Tecnologia Educacional Simbólica. Revista Brasileira de Educação Médica, Rio de Janeiro, v. 29, n. 1, p. 67 80, jan. 2007.

PERRENOUD, P. Novas competências para ensinar. Porto Alegre: Artmed, 2000.

TAKADASHI, Tadao. (Org.). Sociedade da Informação no Brasil: livro verde. Brasília: MCT, 2000.

TEDESCO, Juan Carlos. Educação e novas tecnologias: esperança ou incerteza? São Paulo: Cortez; Co-editor: Escritório UNESCO - Brasília, 2004. Disponível em: http://www.iiep.unesco.org/en/educacao-e-novas-tecnologias-esperanca-ou-incerteza12813. Acesso em: 06 fev. 2022.

VALENTE, José Armando. Integração das tecnologias na educação. Brasília: Ministério da Educação. Secretaria de Educação a Distância, 2008. 
VOSGERAU, D. S. R. A tecnologia na escola: o papel do gestor neste processo. In: BARBOSA, A. F. (Coord). TIC Educação 2011: pesquisa sobre o uso das tecnologias de informação e comunicação nas escolas brasileiras. São Paulo. 2012, p 35-41.

Recebido em: 01/02/2022

Aprovado em: 01/03/2022

Publicado em: 03/03/2022 